\title{
Capacidade funcional de idosos atendidos em um programa do SUS em Belém (PA)
}

\author{
Functional capacity of the elderly attended in a Unified Health \\ System (SUS) program in Belém in the State of Pará
}

Maria Izabel Penha de Oliveira Santos ${ }^{1}$

Rosane Harter Griep ${ }^{2}$

${ }^{1}$ Curso de Enfermagem, Universidade do Estado do Pará. Av. José Bonifácio 1289, Guamá. 66063-010 Belém PA.

princesa50@hotmail.com ${ }^{2}$ Laboratório de Educação em Ambiente e Saúde, Instituto Oswaldo Cruz.

\begin{abstract}
The objective of this study was to evaluate factors associated with functional capacity among the elderly attended in a public health program in Belém in the State of Pará. A sectional study was conducted with 259 elderly people attended in the Hiperdia/SUS program in the period from March to July 2010. The elderly were assessed using the modified physical performance test duly validated in Brazil. Pearson's Chi-square Test, in addition to variance analysis and logistic regression were used for the analysis. The elderly were 68.3 years on average $(S D \pm 6.2)$ and $71 \%$ were female. After adjustments for conflicting variables, it was seen that there were greater chances of worse performance on the test among women, the older and less educated individuals, and those complaining of knee pain and prior diagnosis of cataracts and fair/bad self-assessment of health. Functional capacity is an important health indicator to identify early risks of incapacity and requiring a periodic evaluation, in order to seek care strategies for maintaining the independence of the elderly.
\end{abstract}

Key words The elderly, Gerontology, Public health, Nursing
Resumo O objetivo deste estudo foi avaliar fatores associados à capacidade funcional em idosos atendidos em um programa de saúde pública em Belém (PA). Realizou-se estudo seccional com 259 idosos atendidos no programa Hiperdia/SUS no período de março a julho de 2010. Os idosos foram avaliados pelo teste de desempenho físico modificado, validado no Brasil. Nas análises foram utilizados o teste do qui-quadrado de Pearson, a análise de variância e a regressão. Os idosos tinham em média 68,3 anos $(D P \pm 6,2)$ e 71,0\% eram do sexo feminino. Após ajustes por variáveis de confundimento, observaram-se maiores chances de pior desempenho no teste entre as mulheres, os mais velhos e os de menor escolaridade, além de queixa de dor no joelho, diagnóstico anterior de catarata e autoavaliação de saúde regular/ruim. A capacidade funcional é um indicador de saúde importante para se identificar precocemente riscos de incapacidade e requerendo uma avaliação periódica, no intuito de se buscar estratégias cuidativas para a manutenção da independência dos idosos. Palavras-chave Idoso, Gerontologia, Saúde coletiva, Enfermagem 


\section{Introdução}

Na sociedade atual a assistência à saúde do idoso toma proporção cada vez mais importante. Por um lado, pelo volume aumentado de pessoas a serem atendidas e, de outro, pelo aumento de doenças crônicas e incapacitantes ${ }^{1}$.

No Brasil, a importância relativa do envelhecimento vem ocorrendo de maneira gradual a partir de 1950, e estima-se que, em 2025, o Brasil será o sexto país do mundo quanto ao envelhecimento populacional. Na Região Norte, o índice de envelhecimento ainda é o menor em relação às demais regiões do país; no entanto, tem sido crescente ao longo dos anos. No Estado do Pará, a população de idosos representa $7,1 \%$ da população geral, e a esperança de vida está em torno de 71 anos de idade ${ }^{2-3}$.

O envelhecimento é um processo fisiológico e a manutenção da capacidade funcional no idoso pode ser afetada por diversos fatores. Em geral, os idosos apresentam mais problemas crônicos de saúde do que o restante da população ${ }^{1}$. Dessa forma, a capacidade funcional, em gerontogeriatria, é entendida como o produto da interação entre saúde física, mental, independência nas atividades da vida diária e integração no meio social, tendo como suportes a família e a independência econômica ${ }^{1-6 .}$

$\mathrm{Na}$ medida em que o comportamento das doenças nos diversos segmentos das populações se modifica, medidas de avaliação e/ou indicadores de saúde específicos devem ser utilizados. Assim sendo, a capacidade funcional pode se constituir um importante indicador da saúde e da qualidade de vida, tendo em vista que considera aspectos tais como a autonomia e a independência da pessoa idosa ${ }^{1-4}$. No entanto, observa-se que o uso desse indicador ainda é pouco difundido na rotina dos serviços de saúde, talvez pela falta de difusão do conhecimento gerontológico e geriátrico junto aos profissionais, o que tem contribuído decisivamente para as dificuldades na abordagem global ${ }^{5}$.

$\mathrm{Na}$ Região Norte, e especificamente na cidade de Belém, no Estado do Pará, pesquisas sobre as condições de saúde da população idosa ainda são incipientes. A implantação da Política Nacional de Atenção à Saúde do Idoso ${ }^{4}$, nas Unidades Básicas de Saúde de Belém, ainda não foi efetivamente contemplada.

No contexto atual, as práticas de saúde com o idoso, nessas unidades, ainda estão distanciadas das necessidades reais dessa população, ou seja, são voltadas para a abordagem terapêutica tradicional, dentro de alguns programas já existentes do Ministério da Saúde, como, por exemplo, o Hiperdia-SUS, ou para o encaminhamento a unidades de alta complexidade, como hospitais e unidades de urgência e emergências, fazendo-se de forma inversa às propostas da atenção à saúde.

Dessa forma, o objetivo deste estudo foi avaliar fatores associados à capacidade funcional em idosos atendidos em um programa de saúde pública, em Belém (PA).

\section{Materiais e Métodos}

O tipo de estudo foi epidemiológico seccional, sendo elegíveis idosos cadastrados no Programa Hiperdia-SUS. Os idosos foram recrutados em um Centro de Saúde de Belém (PA) que faz parte do $7^{\circ}$ distrito administrativo da Prefeitura $\mathrm{Mu}-$ nicipal, e os exames de avaliação da capacidade funcional se realizaram em um laboratório especialmente montado para esse fim na Unidade de Ensino e Assistência em Fisioterapia e Terapia Ocupacional. Essas unidades estão localizadas no mesmo espaço geográfico do Centro de Ciências Biológicas e da Saúde (CCBS), da Universidade do Estado do Pará.

Consideraram-se como casos de exclusão do estudo os idosos que necessitassem de algum tipo de ajuda para locomover-se (tais como andador, cadeira de rodas, ou com qualquer outro tipo de impedimento para participar do teste de aptidão funcional); e os que tinham algum tipo de alteração cognitiva observável que dificultasse a compreensão para responder as perguntas do questionário ou que necessitassem que alguém da família respondesse por eles.

Assim, de uma população de referência de 280 idosos cadastrados no programa, 17 foram excluídos por não preencherem os critérios do estudo e quatro recusaram-se a participar. Portanto, integraram o estudo 259 idosos (98,5\% do total de elegíveis), no período de 31/3/2010 até 15/7/2010. Para identificação do número total de pessoas inscritas no programa, consultou-se o sistema de cadastro eletrônico no setor de estatística do Centro de Saúde, ligado à rede do SUS.

$\mathrm{Na}$ estratégia de coleta dos dados, cinco auxiliares de pesquisa receberam treinamento pela pesquisadora sobre todas as etapas do teste, além de serem supervisionados durante sua aplicação. Para tal fim, elaborou-se um manual contendo as descrições necessárias ao desenvolvimento da pesquisa. Destaca-se que todos os resultados do 
teste foram avaliados pela pesquisadora. Os auxiliares da pesquisa eram alunos do último período do curso de graduação em enfermagem.

Considerou-se, para a coleta de dados, o agendamento da consulta mensal dos idosos no programa Hiperdia/SUS, oportunidade em que eles eram convidados a participar do estudo. A triagem inicial era feita pelos examinadores nos prontuários individuais sobre as condições clínicas dos idosos, e os participantes com possibilidade de serem eleitos eram encaminhados para o laboratório onde se realizavam a entrevista e os testes. Nesse momento, os idosos que aceitassem a participar da pesquisa assinavam o Termo de Consentimento Livre e Esclarecido.

O questionário para coleta de dados foi aprimorado em três rodadas de pré-teste, sendo que, nessas etapas, participaram 10 idosos que não compunham a amostra final do estudo. A versão final continha variáveis sociodemográficas (sexo, idade, situação conjugal, escolaridade, fonte de renda, faixa salarial, com quem moravam), informações sobre a prática de atividades físicas e a autoavaliação do estado de saúde ("Comparado a outras pessoas da sua idade, como o Sr.(a) considera o seu próprio estado de saúde?").

Após o preenchimento do questionário, o peso e a estatura dos idosos eram aferidos, segundo técnicas padronizadas para a construção do Índice de Massa Corpórea (IMC). Para avaliação dos dados antropométricos, como peso e altura, estes foram aferidos em uma balança eletrônica digital para pesar pessoas, marca Welmy ${ }^{\circledast}$, modelo W-200A (40x50) ${ }^{\oplus}$, com carga máxima de $200 \mathrm{~kg}$, carga mínima $1 \mathrm{~kg}$, com divisões de 50 g e dimensões da plataforma 390X340 mm, número de série $S 170$, e que foi utilizada especificamente para este estudo.

A balança foi colocada em local plano e o idoso era pesado sem sapatos, agasalhos ou objetos nos bolsos. A estatura foi determinada com régua antropométrica acoplada na própria balança, com capacidade de até $150 \mathrm{~cm}$ e com precisão de $0,2 \mathrm{~mm}$. Além disso, mediu-se a estatura em posição ereta, com a cabeça em plano de Frankfurt, braços estendidos para baixo, pés unidos e encostados ao antropômetro, considerando-se a parte mais alta da cabeça. A haste do antropômetro estando perpendicular ao solo e os ramos perpendiculares à haste.

O Índice de Massa Corporal (IMC) baseouse na classificação da Organização Mundial de Saúde ${ }^{5}$, ou seja, abaixo do peso $(<18,5)$; normal $(18,5-24,9)$; sobrepeso $(25-29,9)$; obeso grau I (30-30,49); obeso grau II (35-39,9); obeso grau
III (>40). Porém essas categorias foram recodificadas para fins de análise em: normal (18,5-24,9); sobrepeso (25-29,9), e obesos, entre os que se classificassem como obesos de grau I, II e III. Quanto aos de baixo peso, não tiveram frequência significativa no estudo, por isso não foram incluídos nessas classes.

Para a medida da pressão arterial, posicionouse o idoso sentado próximo ao examinador, com seu antebraço repousado sobre a mesa. Utilizouse o monitor de pressão arterial automático com braçadeira modelo HEM-742INT ${ }^{\circledast}$, da marca $\mathrm{OMRON}^{\oplus}$. A classificação dos resultados obedeceu ao parâmetro de referência padronizado pelo programa Hiperdia/SUS: Normal < 130/90mmHg; Leve entre 140/90 e 159/99; Moderada entre 160/ 100 e 179/109; e Grave (PS > 180 e PD > 110).

O desfecho - a capacidade funcional - foi avaliado pela versão brasileira do teste de aptidão físico-funcional chamado de Physical Performance Test, ou Teste de Desempenho Físico Modificado (TDFM) ${ }^{6}$. Esse teste avalia habilidades funcionais que simulam a capacidade para realização de atividades cotidianas ou da vida diária, por meio de nove itens, e que é medida em tempo padronizado para realização de cada tarefa, em segundos. A pontuação de cada tarefa varia de zero (incapaz) a 4 pontos, em que este último seria a desejável em um menor tempo possível, e com máxima de 36 pontos, correspondendo esta à melhor performance no teste. Adotou-se a mediana da distribuição para os pontos de corte de desempenho ruim $(<$ mediana) e bom (> mediana).

Para o teste da flexibilidade dos membros superiores, que era avaliada no item de vestir e tirar uma camisa (casaco), foi utilizada uma camisa de algodão branco de manga curta, tamanho "G", para que fosse acessível a todos, e adaptada com tecido de algodão compatível ao clima quente da região amazônica.

Em todas as etapas as recomendações do teste foram obedecidas, destacando-se que a cadeira para avaliar a flexibilidade e a força dos membros inferiores tinha altura de $45 \mathrm{~cm}$ e era sem braços. Para a caminhada de 15 metros foi demarcada, do lado de fora do laboratório, uma superfície plana no solo, sinalizada com fita de cor amarela própria para demarcação de solo, e havia sinais de início e fim da caminhada, sendo o idoso orientado a caminhar com a maior agilidade possível, após o comando do examinador através de um cronômetro.

A escada foi sinalizada do primeiro ao décimo degraus e estes obedeciam à altura de $18 \mathrm{~cm}$, 
como preconiza o TDFM, com corrimãos dos dois lados. Os tempos de desempenho do teste eram cronometrados com um cronômetro profissional Professional stopwatch modelo JS-7062 ${ }^{\circ}$, fabricado por JUNSD-China.

Os dados foram digitados utilizando-se o programa Epi-info (versão 2000), e a análise do banco de dados se realizou através do Statistical Package for Science Services (SPSS, versão 18.0). Foram feitas análises para apresentar a distribuição de frequências por sexo e verificar a associação entre o sexo e a capacidade funcional.

Para a análise univariada das variáveis nominais, utilizaram-se o Teste Qui-quadrado de Pearson e o Teste Exato de Fisher, este quando necessário. As variáveis associadas ao teste de desempenho físico com nível de significância $10 \%$ foram introduzidas em um modelo de regressão logística, para acessar seu efeito independente, permanecendo no modelo final aquelas com significância menor que 5\%. As associações foram estimadas por meio de razão de chances brutas e ajustadas aos respectivos intervalos de $95 \%$ de confiança.

Todos os aspectos éticos em pesquisa foram levados em consideração neste estudo. Os participantes assinaram o Termo de Consentimento Livre e Esclarecido após seu aceite espontâneo à pesquisa, que foi aprovada pelo comitê de ética em pesquisa da EEAN/HESFA/UFRJ.

\section{Resultados}

Os idosos avaliados eram predominantemente do sexo feminino e de faixas etárias mais jovens; na maioria eram casados ou viviam com companheiro(a). Mais da metade deles tinha apenas o nível fundamental incompleto; a principal fonte de renda era a aposentadoria, seguida de pensão, e 74,1\% referiram receber de 1 a 2 salários mínimos. A grande maioria morava com a família (Tabela 1).

Nas análises bivariadas, identificaram-se frequências mais elevadas de desempenho ruim no teste entre as mulheres, entre os mais velhos, entre aqueles sem companheiro(a) e de escolaridade mais baixa. Diversas condições de saúde autorreferidas estiveram associadas ao desempenho; dentre estas, destacamos queixas de dores nos joelhos e nas demais articulações, catarata, dificuldade para dormir, ausência de atividades físicas e autoavaliação de saúde ruim/regular. Não se observou associação entre tabagismo, IMC e níveis pressóricos avaliados (Tabela 2).
Análises ajustadas mostraram que, comparadas aos homens, as mulheres tiveram chances mais elevadas de baixo desempenho no teste (OR $=2,32$; IC95\% = 1,21-4,44). Quanto maior a idade, maiores as chances de desempenho ruim nos testes: respectivamente, $\mathrm{OR}=1,82$ (IC95\% $=0,93$ $3,55)$ e OR $=5,65($ IC95\% $=1,26-25,31)$ para idosos de 71 a 80 anos e 81 a 88 anos. Além disso, os níveis mais baixos de escolaridade se mostraram associados ao desempenho ruim, sendo que, comparados aos de ensino médio, para aqueles que nunca frequentaram a escola as chances eram quase quatro vezes mais elevadas $(\mathrm{OR}=3,92)$, e entre aqueles de ensino fundamental completo ou incompleto as chances foram cerca de duas vezes mais elevadas $(\mathrm{OR}=2,27)$. As queixas de dores no joelho $(\mathrm{OR}=1,73)$ e diagnóstico anterior de catarata $(\mathrm{OR}=1,90)$ se mostraram inde-

Tabela 1. Características sociodemográficas dos idosos do estudo, Belém/PA, $2010(\mathrm{n}=259)$.

\begin{tabular}{|c|c|c|}
\hline Características & $\mathbf{n}$ & $\%$ \\
\hline \multicolumn{3}{|l|}{ Sexo } \\
\hline Masculino & 75 & 29,0 \\
\hline Feminino & 184 & 71,0 \\
\hline \multicolumn{3}{|l|}{ Faixa Etária (anos) } \\
\hline 60 a 70 & 181 & 69,9 \\
\hline 71 a 80 & 63 & 24,3 \\
\hline 81 a 88 & 15 & 5,8 \\
\hline \multicolumn{3}{|l|}{ Situação Conjugal } \\
\hline Casado/Vive com alguém & 134 & 51,7 \\
\hline Solteiro & 39 & 15,1 \\
\hline Viúvo & 59 & 22,8 \\
\hline Divorciado/Separado & 27 & 10,4 \\
\hline \multicolumn{3}{|l|}{ Escolaridade } \\
\hline Nenhuma/não sabe ler & 25 & 9,7 \\
\hline Fundamental incompleto & 137 & 52,9 \\
\hline Fundamental completo & 41 & 15,8 \\
\hline Ensino Médio em diante & 56 & 21,6 \\
\hline \multicolumn{3}{|l|}{ Fonte de Renda } \\
\hline Aposentadoria & 143 & 55,2 \\
\hline Trabalho atual & 25 & 9,7 \\
\hline Pensão & 50 & 19,3 \\
\hline Vive de renda & 4 & 1,5 \\
\hline Não tem renda & 37 & 14,3 \\
\hline \multicolumn{3}{|c|}{ Faixa Salarial em Salários mínimos* } \\
\hline$<1$ & 13 & 5,2 \\
\hline 1 a 2 & 186 & 74,1 \\
\hline 3 ou + & 52 & 20,7 \\
\hline \multicolumn{3}{|l|}{ Mora atualmente } \\
\hline Com a família & 229 & 88,4 \\
\hline Sozinho & 28 & 10,8 \\
\hline Com outras pessoas & 2 & 0,8 \\
\hline
\end{tabular}

*Salário mínimo na época de R \$ 520,00 
Tabela 2. Descrição de variáveis segundo o teste de desempenho físico modificado (TDFM) (n = 259).

\begin{tabular}{|c|c|c|c|}
\hline \multirow[b]{2}{*}{ Variáveis } & \multicolumn{2}{|c|}{ Desempenho no teste } & \multirow[b]{2}{*}{ Valor de $p$} \\
\hline & $\begin{array}{c}\text { Melhor } \\
\text { n }(\%)\end{array}$ & $\begin{array}{l}\text { Pior } \\
\text { n (\%) }\end{array}$ & \\
\hline \multicolumn{4}{|l|}{ Variáveis sociodemográficas } \\
\hline Sexo & & & $<0,001$ \\
\hline Masculino & $54(72,0)$ & $21(28,0)$ & \\
\hline Feminino & $87(47,3)$ & $97(52,7)$ & \\
\hline Faixa etária (anos) & & & 0,001 \\
\hline 60 a 70 & $111(61,3)$ & $70(38,7)$ & \\
\hline 71 a 88 & $30(38,5)$ & $48(61,5)$ & \\
\hline Tem companheiro(a) & & & 0,001 \\
\hline Sim & $86(64,2)$ & $48(35,8)$ & \\
\hline Não & $55(44,0)$ & $70(56,0)$ & \\
\hline Escolaridade & & & 0,001 \\
\hline Ensino médio completo & $42(75,0)$ & $14(25,0)$ & \\
\hline Ensino médio incompleto & $23(56,1)$ & $18(43,9)$ & \\
\hline Ensino fundamental & $69(50,4)$ & $68(49,6)$ & \\
\hline Não frequentou escola & $7(28,0)$ & $18(72,0)$ & \\
\hline \multicolumn{4}{|l|}{ Condições de saúde autorreferidas } \\
\hline Dor no joelho & & & 0,008 \\
\hline Sim & $65(46,8)$ & $74(53,2)$ & \\
\hline Não & $76(63,3)$ & $44(36,7)$ & \\
\hline Dor em outras articulações & & & 0,010 \\
\hline $\operatorname{Sim}$ & $72(47,7)$ & $79(52,3)$ & \\
\hline Não & $69(63,9)$ & $39(36,1)$ & \\
\hline Catarata & & & $<0,0001$ \\
\hline Sim & $45(39,5)$ & $69(60,5)$ & \\
\hline Não & $96(66,2)$ & $49(33,8)$ & \\
\hline Dificuldades para dormir & & & 0,006 \\
\hline Sim & $49(44,5)$ & $61(55,5)$ & \\
\hline Não & $92(61,7)$ & $57(38,3)$ & \\
\hline Tabagismo & & & 0,342 \\
\hline Fumante atual & $7(77,8)$ & $2(22,2)$ & \\
\hline Ex-fumante & $72(54,4)$ & $60(45,5)$ & \\
\hline Nunca fumou & $62(52,5)$ & $56(47,5)$ & \\
\hline Uso de bebidas alcoólicas & & & 0,055 \\
\hline Nunca & $97(50,5)$ & $95(49,5)$ & \\
\hline Raramente & $33(62,3)$ & $20(37,7)$ & \\
\hline Frequentemente & $11(78,6)$ & $3(21,4)$ & \\
\hline Faz atividades físicas de lazer & & & 0,025 \\
\hline Sim & $77(61,6)$ & $48(38,4)$ & \\
\hline Não & $64(47,8)$ & $70(52,2)$ & \\
\hline Autoavaliação da saúde & & & 0,002 \\
\hline Muito boa/Boa & $90(62,9)$ & $53(37,1)$ & \\
\hline Regular/Ruim & $51(44,0)$ & $65(56,0)$ & \\
\hline \multicolumn{4}{|l|}{ Condições avaliadas } \\
\hline IMC & & & 0,202 \\
\hline Normal/baixo peso & $32(50,8)$ & $31(49,2)$ & \\
\hline Sobrepeso & $74(60,2)$ & $49(39,8)$ & \\
\hline Obeso & $35(47,9)$ & $38(52,1)$ & \\
\hline Níveis pressóricos & & & 0,174 \\
\hline$\leq 140 \times 90 \mathrm{mmHg}$ & $31(60,8)$ & $20(39,20)$ & \\
\hline$<159$ x 99mmHg & $64(54,7)$ & $53(45,3)$ & \\
\hline De 160 x 100 a $179 \times 109 \mathrm{mmHg}$ & $36(57,1)$ & $27(42,9)$ & \\
\hline$\geq 180 \times 101 \mathrm{mmHg}$ & $10(35,7)$ & $18(64,3)$ & \\
\hline
\end{tabular}


pendentemente associadas ao desempenho ruim no teste, além da autoavaliação de saúde regular/ $\operatorname{ruim}(\mathrm{OR}=2,01)$ (Tabela 3$)$.

\section{Discussão}

Identificaram-se no estudo desvantagens das mulheres em relação à capacidade funcional avaliada. Diferenças de gênero devem ser destacadas quando se aborda o envelhecimento. Nesse contexto, as mulheres, por viverem cerca de 8 a10 anos mais do que os homens, apresentam maior vulnerabilidade às doenças crônicas não transmissíveis (DCNT) e às hospitalizações, o que implica em necessidades distintas de cuidados e ainda as coloca com maior chance de ficarem viúvas e em situação socioeconômica desvantajosa $^{6-8}$.

Assim como outros estudos brasileiros envolvendo idosos ${ }^{9-11}$, identificou-se predominância feminina. Embora o aumento da esperança de vida ao nascer se observe para ambos os sexos, a taxa de sobrevida das mulheres se mantém superior à dos homens, considerando-se os desníveis regionais ${ }^{2-8}$. Além disso, as mulheres reconhecidamente buscam mais os serviços de saú$\mathrm{de}^{9}$, fato que também pode explicar o maior nú- mero de mulheres, já que o estudo abrangeu um programa específico da Unidade de Saúde.

A escolaridade dos idosos deste estudo foi baixa, constituindo-se em uma condição social desfavorável para eles, já que tem influência no acesso aos serviços de saúde, em oportunidades de participação social e na compreensão de seu tratamento e do seu autocuidado, entre outros. Cabe destacar que essa situação reflete as diferenças regionais do país. Embora se tenha avançado nos níveis de escolaridade no período de 1998 a 2008, entre essa faixa etária no Brasil, os nortistas apresentaram, em média, três anos de estudo e os nordestinos uma média ainda mais baixa $(2,7 \text { anos })^{9-12}$.

Aliadas à baixa escolaridade observaram-se desvantagens socioeconômicas. Embora reconhecendo a limitação do presente estudo no que se refere à generalização dos achados, outros estudos apontaram idosos em condições semelhantes em outras regiões economicamente mais desenvolvidas em relação ao Pará. Em um estudo realizado em Porto Alegre, 21,4\% dos idosos da amostra tinham condição financeira aproximada à realidade dos deste estudo ${ }^{13}$. Em Juiz de Fora, Minas Gerais, cerca de $82,9 \%$ dos idosos tinham como renda mensal dois salários mínimos e $14 \%$ moravam sozinhos ${ }^{14}$. Um perfil soci-

Tabela 3. Associações brutas e ajustadas do sexo e baixo desempenho do teste TDFM dos idosos do estudo, Belém/PA, 2010.

\begin{tabular}{|c|c|c|}
\hline Variáveis & Razão de Chances brutas & Razão de chances ajustadas \\
\hline \multicolumn{3}{|l|}{ Sexo } \\
\hline Masculino & 1,0 & 1,0 \\
\hline Feminino & $2,86(1,60-5,13)$ & $2,32(1,21-4,44)$ \\
\hline \multicolumn{3}{|l|}{ Faixa etária (anos) } \\
\hline 60 a 70 & 1,0 & 1,0 \\
\hline 71 a 80 & $2,11(1,18-3,78)$ & $1,82(0,93-3,55)$ \\
\hline 81 a 88 & $6,34(1,73-23,27)$ & $5,65(1,26-25,31)$ \\
\hline \multicolumn{3}{|l|}{ Escolaridade } \\
\hline Ensino médio ou mais & 1,0 & 1,0 \\
\hline Ensino fundamental completo & $2,35(0,99-5,57)$ & $2,27(0,88-5,83)$ \\
\hline Ensino fundamental incompleto & $2,95(1,48-5,90)$ & $2,28(1,09-4,77)$ \\
\hline Não frequentou escola & $7,71(2,66-22,31)$ & $3,92(1,22-12,64)$ \\
\hline \multicolumn{3}{|l|}{ Dor no joelho } \\
\hline Não & 1,0 & 1,0 \\
\hline Sim & $1,97(1,19-3,24)$ & $1,73(0,99-3,04)$ \\
\hline \multicolumn{3}{|l|}{ Catarata } \\
\hline Não & 1,0 & 1,0 \\
\hline Sim & $2,01(1,22-3,31)$ & $1,90(1,07-3,36)$ \\
\hline \multicolumn{3}{|l|}{ Autoavaliação de saúde } \\
\hline Ótima/Boa & 1,0 & 1,0 \\
\hline Regular/Ruim & $1,97(1,19-3,24)$ & $2,01(1,15-3,51)$ \\
\hline
\end{tabular}


oeconômico semelhante foi descrito em estudo em Goiânia, entre os cadastrados na Estratégia Saúde da Família ${ }^{15-24}$.

No Brasil a proporção de idosos que moravam com filhos era de 33,3\% na última Pesquisa Nacional por Amostra de Domicílios, e nas Regiões Norte e Nordeste esse percentual era bem mais elevado, mais de $50 \%$ se encontravam nessa condição ${ }^{16}$. Neste estudo, uma grande proporção referiu morar com a família e, na maioria, os filhos estavam incluídos. Destacando-se que a família tem um importante papel no apoio social e afetivo para as pessoas que envelhecem, em especial no atendimento de suas necessidades.

A presença de dor tem sido citada como um fator limitante nos idosos, em testes de desempenho de aptidão funcional, o que se reflete nas suas atividades cotidianas ${ }^{7-17}$. Esse fato, talvez somado às disfunções visuais relatadas pelas mulheres deste estudo, pode ter influenciado num desempenho menor delas no teste global de aptidão física, em relação aos homens.

Quanto à presença de doenças crônicas, estas representam no Brasil a maior carga de doença e de causa de morte, entre elas as cardiovasculares e o diabetes do tipo 2. As DCNT representaram no ano de 2007 cerca de $72 \%$ das mortes, com peso relativo maior para adultos e idosos. Associado a esse fator, tem-se a epidemia da obesidade, que concorre para o aumento da prevalência da hipertensão arterial e diabetes, tornando-se um dos maiores desafios para a saúde brasileira atual ${ }^{11-18}$.

Em estudo que avaliou o IMC em pessoas idosas e associou-o com o Índice de Aptidão Funcional Global (IAFG), essa relação encontrou que $90,4 \%$ das mulheres com excesso de peso tiveram esse índice fraco em relação às com peso normal $(\mathrm{p}<0,01)^{15-18}$. No presente estudo, apesar do IMC não ter tido associação com o desempenho no teste de aptidão, é uma variável de considerável importância em termos de saúde pública, já que reflete transformações sociais ocorridas ao longo do tempo: aumento do sedentarismo e maior acesso a alimentos, além das mudanças de hábitos nutricionais, muitas vezes não saudáveis. Trata-se de uma variável que expõe a população a um risco maior de doenças crônicas como o diabetes e a hipertensão arterial, predispondo a alterações da capacidade funcional dos que envelhecem, no Brasil ${ }^{11}$.

A capacidade funcional pode se constituir em uma medida útil na identificação precoce de futuras incapacidades, levando em conta características fisiológicas, forma de evolução da doença e adesão ao tratamento da hipertensão e diabetes ${ }^{10-}$
${ }^{15}$, que podem se dar, também, de forma diferenciada entre homens e mulheres, e isso contribuiria para direcionar ao planejamento de ações adequadas às reais necessidades para cada grupo.

No presente estudo identificaram-se diferenças significativas entre homens e mulheres no desempenho dos testes que compõem a avaliação da capacidade funcional, o que pode ser explicado por diferenças fisiológicas e anatômicas apresentadas pelo grupo de idosos, quando avaliados. Alguns estudos ${ }^{20-21}$ citam que os ligamentos e músculos das mulheres são mais elásticos e flexíveis que os dos homens, devido à menor densidade dos tecidos. Assim como a sua flexibilidade é influenciada pela melhor e mais rápida capacidade de descontração do músculo e pelo maior comprimento da coluna vertebral lombar; no entanto, a presença de doença crônica (entre elas, a hipertensão arterial) e dores concorrem para limitar essa flexibilidade $\mathrm{e}^{22-25}$.

Além disso, o uso de certos anti-hipertensivos, tais como os beta-bloqueadores, é capaz de reduzir a resposta cardíaca aos exercícios, e esse efeito pode limitar a capacidade de trabalho físico durante atividades que exigem débito cardíaco alto, como, por exemplo, subir escadas, e a prática de exercícios, entre outros. Esse fato também pode ter influenciado para os idosos não terem alcançado escores mais altos nessa tarefa, principalmente entre as mulheres ${ }^{23}$.

O teste de pegar no chão uma moeda com os dedos polegar e indicador indica um ato motor fino preservado da musculatura da mão, o que reflete a capacidade na atividade diária de ser capaz de abrir a tampa de um refrigerante, ou de uma lata, por exemplo. Já, nas tarefas que exigiram maior agilidade, como caminhar 15 metros, e força muscular maior no quadríceps, como subir um lance de escada, os homens deste estudo tiveram melhor desempenho do que as mulheres.

Com relação à força e capacidade dos membros inferiores, em um estudo com idosos os autores enfatizam que a habilidade de levantarse de uma cadeira ou da cama, embora seja considerada simples, é uma ação de complexa que pode estar relacionada a desordens músculo-esqueléticas e neuromotoras, o que exigiria muito do idoso ${ }^{25}$.

É importante destacar algumas limitações referentes aos achados do presente estudo. Nesse aspecto, destacamos a generalização limitada aos idosos de maneira geral, já que o estudo tratou de um grupo em tratamento para doenças crônicas de uma unidade de saúde. A comparação dos achados do presente estudo foi limitada em 
função de que os estudos semelhantes ${ }^{7-17}$ avaliaram a capacidade funcional apenas de mulheres idosas.

\section{Considerações finais}

Em síntese, os resultados do presente estudo mostraram que entre os idosos avaliados, o pior desempenho no teste de avaliação da capacidade funcional ocorreu entre as mulheres, os mais velhos e aqueles de menor escolaridade, além de aspectos relacionados à saúde, tais como queixa de dor nos joelhos, diagnóstico de catarata e autoavaliação de saúde ruim/regular. Embora alguns desses fatores estejam relacionados ao envelhecimento fisiológico, os resultados sugerem que a rede causal relacionada ao declínio da capacidade funcional é multifatorial e complexa.

Portanto, a escuta atenta às queixas dos idosos, o reconhecimento de fatores associados com a incapacidade, a atenção aos sinais precoces de limitações e a busca de estratégias efetivas de pre- venção e de reabilitação podem contribuir para um envelhecimento sem muitas perdas funcionais. A postergação da incapacidade poderia diminuir a carga em saúde coletiva, já que pode gerar necessidade de internações mais frequentes, custos com medicamentos, um cuidador que acompanhe as atividades diárias e alterações na dinâmica familiar para o atendimento das necessidades.

A avaliação da capacidade funcional de pessoas idosas pelos serviços de saúde no Brasil é uma recomendação da Política Nacional de Atenção à Saúde do Idoso ${ }^{5}$. Segundo esta, a avaliação deve ser feita de forma integrada e envolvendo diferentes profissionais de saúde Assim, é relevante que a equipe multiprofissional e interdisciplinar envolvida possa desenvolver ações que promovam o redirecionamento da prática assistencial, na perspectiva de oferecerem um cuidado em saúde voltado à sua realidade e naquilo que os idosos de fato apresentam como necessidades, no intuito de que promovam um cuidado efetivo, na aplicação de tecnologias e na promoção da saúde.

\section{Colaboradores}

MIPO Santos trabalhou na pesquisa de campo, na metodologia e na redação final do artigo, e RH Griep, na análise estatística e orientação final. 


\section{Referências}

1. Veras R, Lourenço R, Martins CSF, Sanches MAS, Chaves PH. Novos paradigmas do modelo assistencial no setor saúde: conseqüência da explosão populacional dos idosos no Brasil. In: Veras R. Terceira Idade: gestão contemporânea em saúde. Rio de Janeiro: Relume-Dumará, UNATI/UERJ; 2002. p. 11-81.

2. Instituto Brasileiro de Geografia Estatístico (IBGE). Anuário Estatístico. Rio de Janeiro: IBGE; 2000.

3. Giacomin K. Avaliação funcional: o que é função, como avaliar e como lidar com perdas. In: Saldanha AL, Caldas CP, organizadores. Saúde do idoso: a arte de cuidar. Rio de Janeiro: Interciência; 2004. p. 132-142.

4. Brasil. Portaria GM 399 de 22 de fevereiro de 2006. Divulga o Pacto pela Saúde 2006 - Consolidação do SUS e aprova as Diretrizes Operacionais do Referido Pacto. Diário Oficial da União 2006; fev 23.

5. World Health Organization (WHO). Physical status: the use and interpretation of anthropometry. Geneve: Report of a WHO Expert Committee; 1995.

6. Mitre NCD, Dias RC, Dias JMD, Faria APS, Costa DC, Carvalho GM, Ribeiro ACP. Adaptação para o português e confiabilidade de uma versão modificada do physical performance test. Geriatr Gerontol 2008; 2(3):104-119.

7. Brasil. Ministério da Saúde (MS). Cadernos de atenção básica: saúde do idoso. Brasília: MS; 2006.

8. Camarano AA. Envelhecimento da população brasileira: uma contribuição demográfica. In: Freitas, EV, organizador. Tratado de gerontologia e geriatria. Rio de Janeiro: Guanabara Koogan; 2006. p. 88-105.

9. Instituto Brasileiro de Geografia e Estatística (IBGE). Sintese de indicadores sociais: uma análise das condições de vida da população brasileira. Rio de Janeiro: IBGE; 2008.

10. Lebrão ML, Laurenti R. Saúde, bem-estar e envelhecimento: o estudo sabe no Município de São Paulo. Rev Bras Epidemiol 2000; 8(2):127-141.

11. Schmidt MI, Duncan BB, Silva, GA, Menezes AM, Monteiro CA, Barreto SM, Chor D, Menezes PR. Doenças crônicas não transmissíveis no Brasil: carga e desafios atuais. [acessado $2013 \mathrm{fev} 23$ ]. Disponível em: http://download.thelancet.com/flatcontentassets/ pdfs/brazil/brazilpor4.pdf

12. Souza LM, Morais EP, Barth QCM. Características demográficas, socioeconômicas e da saúde dos idosos de um programa de saúde da família de Porto Alegre, Brasil. Rev Latamericana Enferm 2006; 14(6):901-906

13. Alves MJM, Ribeiro LC, Milane JL, Almeida AM. Perfil da capacidade funcional do idoso. In: Anais do Congresso Nacional de Estudos Populacionais; 2008; Caxambu, MG. p. 1-18.

14. Costa EC, Nakatani AYK, Bachion MM. Capacidade dos idosos da comunidade para desenvolver atividades da vida diária e atividades instrumentais da vida diária. Acta Paul Enferm 2006; 19(1):43-48.

15. Instituto Brasileiro de Geografia Estatística (IBGE). Sintese de indicadores sociais: uma análise das condições de vida da população brasileira. Rio de Janeiro: IBGE; 2009.
16. Coelho MAGM. A influência da atividade física na dor crônica e na capacidade funcional em idosos comunitários de Itaúna-MG [dissertação]. Belo Horizonte: Universidade Estadual de Minas Gerais; 2009.

17. Fielder MM, Peres KG. Capacidade funcional e fatores associados em idosos do sul do Brasil: um estudo de base populacional. Cad Saude Publica 2008; 24(2):409-415.

18. Mastroeni MF, Erzinger GS, Mastoeni SSBS, Silva NN, Marucci MFN. Perfil demográfico de idosos da cidade de Joinville, Santa Catarina: estudo de base domiciliar. Rev Bras Epidemiol 2000; 10(2):2132.

19. Ueno LM, Okuma SS, Miranda ML, Jacob Filho W. Análise dos efeitos quantitativos e qualitativos de um programa de educação física sobre a flexibilidade do quadril em indivíduos com mais de 60 anos. Motriz 2000; 6(1):9-16.

20. Mazo GZ, Kulkamp W, Lyra VB, Prado APM. Aptidão funcional geral e índice de massa corporal de idosas praticantes de atividade física. Rev Bras Cineantropom Desempenho Hum 2006; 8(4):46-51.

21. Mazo GZ, Benedetti TRB, Gobbi S, Ferreira L, Lopes MA. Valores normativos e aptidão funcional em homens de 60 a 90 anos de idade. Rev Bras Cineantropom Desempenho Hum 2010; 12(5):316-323.

22. Ciccone CD. Considerações farmacológicas para idosos. In: Ciccone CD. Manual de reabilitação geriátrica. Rio de Janeiro: Guanabara Koogan; 2001. p. 47-52.

23. Nunes DP, Nakatani AYK, Silveira EA, Bachion MM, Souza MR. Capacidade funcional, condições socioeconômicas e da saúde de idosos atendidos por equipe de saúde da família de Goiânia. Cien Saude Colet 2010; 15(6):144-150.

24. Parahyba MI, Veras RP. Diferenciais sociodemográficos no declínio funcional em mobilidade física entre idosos no Brasil. Cien Saude Colet 2008; 13(4):1257-1264.

25. Camara G, Gerez AG, Velardi MLJMM. Capacidade funcional do idoso: formas de avaliação e tendências. Acta Fisiátrica 2008; 15(4):249-256.

Artigo apresentado em 10/07/2011

Aprovado em 15/08/2011

Versão final apresentada em 13/09/2011 
